\title{
Synovial sarcoma of the extremities and trunk: a long-lasting disease
}

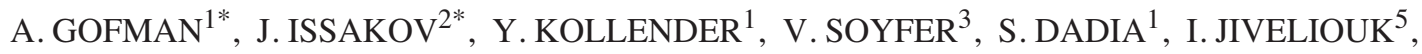 \\ G. FLUSSER $^{6}$, J. BICKELS ${ }^{1}$, I. MELLER ${ }^{1}$ and O. MERIMSKY ${ }^{4}$ \\ ${ }^{1}$ National Unit of Orthopedic Oncology; ${ }^{2}$ Unit of Bone and Soft Tissue Pathology, Department of Pathology; Units of \\ ${ }^{3}$ Radiation Therapy, and ${ }^{4}$ Bone and Soft Tissue Oncology, ${ }^{5}$ Division of Oncology; ${ }^{6}$ Unit of Skeletal Radiology, \\ Department of Radiology, Tel-Aviv Sourasky Medical Center, affiliated with the Sackler School \\ of Medicine, Tel-Aviv University, 6 Weizmann Street, Tel-Aviv 64239, Israel
}

Received April 25, 2007; Accepted July 20, 2007

\begin{abstract}
Synovial sarcoma (SS) of an extremity or trunk is relatively rare and is approached by limb sparing surgery (LSS), radiation therapy (RT) and chemotherapy. We conducted a retrospective analysis of the clinical and histopathological data of 73 patients with proven SS. At a median follow-up time of 6 years, local recurrence was seen in 17.8 and systemic recurrence $35.6 \%$ of patients (local-only, 6.8; systemic-only, 24.6; combined, $11 \%$ ). The 10-year local recurrence-free survival (LRFS), systemic recurrence-free survival (SRFS) and overall survival (OS) rates were 78, 68 and 61\%, respectively. LRFS was significantly better in patients treated with isolated limb perfusion (ILP); SRFS was influenced by the delay until diagnosis. The practical aspects of our observations are the need for long-term follow-up in order to diagnose recurrences, the fact that not all local or distant recurrences are necessarily associated with a shortening of OS time and the important role of induction ILP with TNF in cases of extremity SS.
\end{abstract}

\section{Introduction}

Synovial Sarcoma (SS), part of a rare group of mesenchymal tumors, comprises $8-10 \%$ of all cases of soft tissue sarcomas (STSs) and accounts for $1 \%$ of all malignancies. SS most commonly affects adolescents and young adults (1). Approximately $80 \%$ of SSs occur in the extremities, mostly in the lower limb followed by the upper limb and trunk (2).

Correspondence to: Dr Ofer Merimsky, Unit of Bone and Soft Tissue Oncology, Division of Oncology, Tel-Aviv Sourasky Medical Center, affiliated with the Sackler School of Medicine, Tel-Aviv University, 6 Weizmann Street, Tel-Aviv 64239, Israel

E-mail: oferm@tasmc.health.gov.il

${ }^{*}$ Contributed equally.

Key words: synovial sarcoma, surgery, radiation therapy, isolated limb perfusion
SS is a high-grade STS with features of epithelial differentiation showing a wide range of morphological appearances. There are several histological variants including biphasic (the classic form with mixed epithelial and spindle cell components), monophasic (predominantly epithelial or fibrous), poorly differentiated, cystic, myxoid, calcifying and bone/cartilage forming (3). Although the tumor is named 'synovial' sarcoma because of its histologic resemblance to normal synovial tissue, intra-articular SS is a rare occurrence and its exact histogenesis or line of differentiation remains unclear (3). Several authors claim that SSs are in fact STSs that aberrantly express epithelial markers such as cytokeratin (CK) and epithelial membrane antigen (EMA) (4). The differential diagnosis of non-biphasic SS includes malignant peripheral nerve sheath tumor, malignant fibrous histiocytoma (MFH), fibrosarcoma and leiomyosarcoma. Poorly differentiated SS can imitate small round cell tumors such as Ewing's sarcoma and peripheral neuroectodermal tumor (5).

Translocation, involving chromosomes $\mathrm{X}$ and $18[\mathrm{t}(\mathrm{X} ; 18)$ (p11;q11)], is present in more than $90 \%$ of SSs (6). Cloning of the DNA adjacent to the breakpoints of the $t(X ; 18)$ results in the fusion of the SYT gene at 18q11.2 to either of two genes, SSX1 or SSX2, at Xp11.2. This rearranged configuration of DNA, called SYT-SSX 1 and SYT-SSX 2 transcripts, is transcribed to the mRNA and provides a specific marker for SS which can be detected by RT-PCR testing $(7,8)$.

SS shows two specific clinical-radiological characteristics. Except for the appearance of spotty calcifications throughout the tumor mass in about $30 \%$ of cases (5), it is radiologically no different than other STSs and should be included in the differential diagnosis of cystic soft tissue masses (9). Although most STSs spread in hematogenous way, lymphatic spread may occur in SS and is responsible for lymph node involvement in about $15 \%$ of cases (3).

The management of SS, as well as other STSs, is based on a multidisciplinary approach towards limb sparing. While surgery and post-operative radiation therapy (RT) are proven modalities of treatment, pre- and post-operative chemotherapy remain investigational (10). Amputation surgery is reserved for locally advanced cases or for palliation. Isolated limb perfusion (ILP) with TNF $\alpha$ and melphalan has been 
suggested for limb salvage or pre-operative therapy in selected patients (11).

The present report summarizes 15 -years' experience using a multidisciplinary approach in 73 consecutive patients with SS that were referred to our center. Retrospective analysis of the clinical, surgical and pathological data yielded several important conclusions.

\section{Patients and methods}

A retrospective analysis of the clinical and histopathological data of 73 consecutive patients with proven SS, treated at the National Unit of Orthopedic Oncology from January 1991 to December 2004, was performed. In each case, all surgical, medical and oncological files were retrieved and reviewed, and all available microscopic slides were re-inspected in order to verify the diagnosis. Information on age at time of diagnosis, date of diagnosis, sex, histopathological diagnosis and clinical group, and the details of therapy and clinical outcome, were available for all patients.

Statistical analysis included descriptive statistics, the two-by-two test and Kaplan-Meier survival analysis, which were applied for statistical work-up using Microsoft Excel and GraphPad Prism 4 software. Overall survival (OS) was calculated from the date of tissue diagnosis until death. Only disease-specific death events were considered. Diseasefree survival (DFS) was calculated separately for systemic recurrence-free survival (SRFS) and local recurrence-free survival (LRFS) from the date of definitive surgery to the date of an event (LR or metastases). OS and DFS (SRFS or LRFS) were estimated using the Kaplan-Meier method.

All patients in this series were treated and followed by one team as follows: new patients with soft tissue masses suspected to be STS underwent clinical and radiological evaluation prior to biopsy; a tumor specimen for histological diagnosis was obtained by computed tomography-guided core needle biopsy, open incisional biopsy or open excisional biopsy; specimens taken in other medical centers were obtained for revision; the AMSTS (Enneking's) staging system was applied for describing clinical and surgical disease status (12).

The diagnosis of STS and the determination of sarcoma subtypes was made by a dedicated sarcoma-pathologist. Paraffin sections $(7 \mathrm{~mm}$ ) were used for light microscopy and for immunohistochemical staining to detect the presence of various antigens: reticulin, mucicarmine, alcian blue (with and without hyaluronidase digestion), CK 5, 6, 7, 8, 18, 19, 20, vimentin, EMA, S-100, CEA, CD34, CD 99, calretinin, Bcl-2, Leu-7, muscle-specific actin, desmin and keratin. Cytogenetic studies for synovial sarcoma-related specific changes, i.e. $\mathrm{t}(\mathrm{X} ; 18)(\mathrm{p} 11 ; \mathrm{q} 11)$, were performed on fresh tissue samples. The French Federation of Cancer Centers grading system was used for tumor characterization according to differentiation, mitotic rate and tumor necrosis. Grading was determined as low- (G1), intermediate- (G2) or high-grade (G3).

Pre-operative therapy was considered only for patients with high-grade IIB lesions adjacent to the neurovascular bundle or bone and no serious systemic illness. Doxorubicin-based chemotherapy with ifosfamide was administered for 2-4 cycles of 21 days. Limb sparing surgery (LSS) was performed 2-3 weeks after the end of the last chemotherapy cycle. Adjuvant chemotherapy consisted of additional cycles of ADR + IFX, for up to a total of 6 cycles, only if a favorable pathological response to chemotherapy was observed (10).

Patients with high-grade IIB lesions adjacent to the neurovascular bundle or bone, especially in the lower limb, considered to be poor candidates for systemic induction chemotherapy due to co-morbidities, were referred to ILP with recombinant tumor-necrosis factor $\alpha(\mathrm{rTNF} \alpha)$ and melphalan. Briefly, a bolus of $4 \mathrm{mg} \mathrm{TNF} \alpha$ was injected into an isolated limb followed by a $1.5 \mathrm{mg} / \mathrm{kg}$ body-weight bolus injection of melphalan, while the limb temperature was kept at $39-40^{\circ} \mathrm{C}$ (11). LSS was carried out 6-8 weeks later.

Pre-operative evaluation included an MRI of the involved extremity, chest CT scan, bone scan, MUGA scan and blood count and chemistry.

LSS was attempted in all the cases by the same surgical team. Fresh specimens were obtained for pathological examination. Tumor size, surgical margins and the presence of tumor necrosis, hemorrhage, cyst formation, fibrosis or calcifications were studied. In cases of LSS after neo-adjuvant therapy (ILP or chemotherapy), two slices from the largest diameter of the tumor mass (maximal sagital and tangential cross sections) were entirely embedded in paraffin and histologically examined using mapping technique. Surgical margins were defined as adequate (R0) in every case with negative microscopic margins and a normal tissue cuff of $1 \mathrm{~cm}$ or more. Inadequate $(\mathrm{R} 1)$ resection was defined as resection at close or positive margins.

RT was delivered following chemotherapy through a linear accelerator photon beam to the target volume by 2 opposed fields. A midplane dose of 63 or 70 Gy was administered in cases of marginal excision or involved margins. RT details have been reported elsewhere (13).

Follow-up was carried out by a multidisciplinary team and included medical details, physical examination, blood count, serum biochemistry and chest plain film every 3 months, and chest and limb CT or MRI every 6 months, for the first 2 years. Over subsequent years, follow-up was carried out biannually and, after the 5 th year, once annually. The purpose of the extensive follow-up protocol for all STSs was to diagnose resectable and potentially curable metastases or late relapses and complications, to assess rehabilitation and to screen for second primary malignancies.

\section{Results}

A total of 73 patients (46 male and 29 female) were treated over 15 years. The follow-up time was 2-15 (median 6) years. The median age at time of diagnosis was 38 (range 8-82 years). Four patients were younger than 15 and 9 were older than 60 years. Of the 73 patients, 41 were primarily diagnosed in our unit and 32 were diagnosed elsewhere and referred to us for treatment.

The elapsed time between the first appearance of a mass or the first symptoms and the establishment of the correct diagnosis was between 2 and 29 (median 18) months. There were 62 new and untreated cases and 11 cases which were referred with local recurrence or systemic relapse after having been treated elsewhere. The majority of the primary 


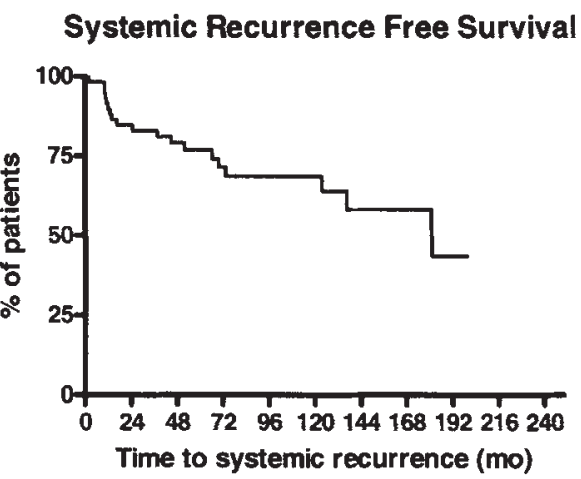

Accumulation of Systemic Recurrences

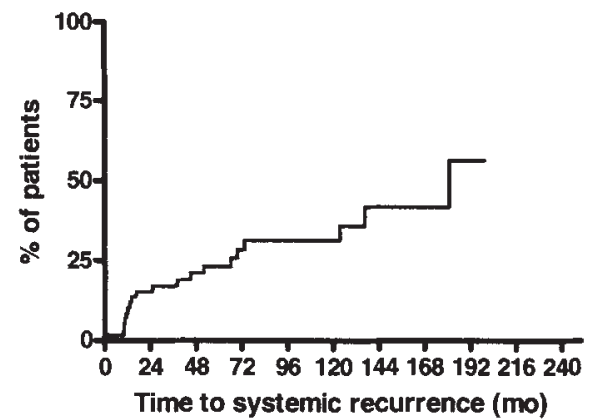

Local Recurrence Free Survival

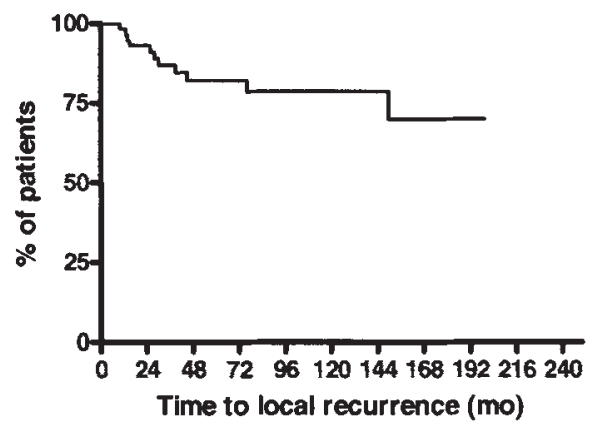

Accumulation of Local Recurrences

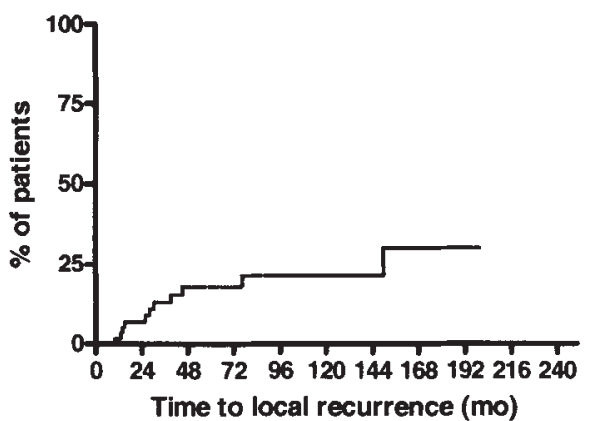

Overall Survival

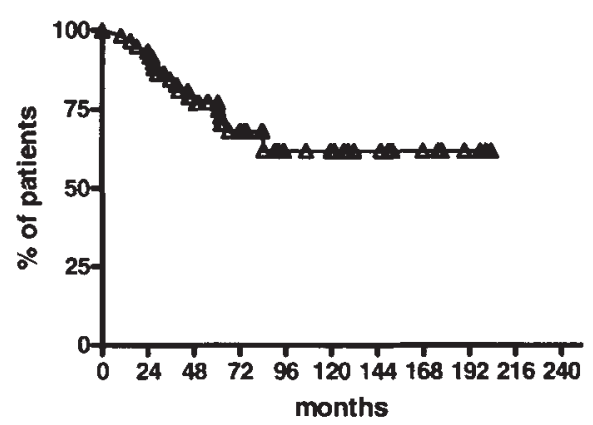

Figure 1. Local recurrence-free survival, systemic recurrence-free survival, accumulation of events and OS of patients with synovial sarcoma.

tumors $(80 \%)$ originated in an extremity (arm, 2 cases; forearm 7; hand 3; thigh 15; knee including poplyteal fossa, 10 ; leg 8 ; foot 13 ), while only $20 \%$ originated in the trunk (neck 2; shoulder 3; groin 6; pelvis 3). In 19 patients the tumor originated in true extra-compartmental sties: axilla, groin and poplyteal fossa. In another 2 patients the tumor was located intra-articularly.

Of the patients, $48(65.7 \%)$ had tumors $<5 \mathrm{~cm}$ in diameter, $17(22.3 \%)$ had tumors between $5-10 \mathrm{~cm}$ in diameter and 8 $(12 \%)$ had tumors $>10 \mathrm{~cm}$ in diameter.

Tumor stage was AMSTS IIB in 62 patients and IIIB in 11 patients at presentation. In our series no case presented with lymph node involvement. For statistical analysis tumor size was marked as $\mathrm{T} 1(\leq 5 \mathrm{~cm})$ or $\mathrm{T} 2(>5 \mathrm{~cm})$. The most common histological subtype was monophasic (37 cases) followed by biphasic (32 cases) and poorly differentiated (4 cases). All tumors were considered high-grade. Cytogenetic analysis was carried out in 12 patients and, in all cases, a $\mathrm{t}(\mathrm{X} ; 18)(\mathrm{p} 11 ; \mathrm{q} 11)$ translocation was found.

Induction ILP was performed on 10 patients and achieved tumor necrosis of $\geq 90 \%$ in 7 of them (median degree of necrosis $90 \%$ ). Pre-operative followed by post-operative chemotherapy was administered in 21 cases $(28 \%)$, and achieved tumor necrosis of $\geq 90 \%$ in 6 patients (median tumor necrosis $40 \%$ ). Post-operative-only chemotherapy was administered to 7 patients. 
The surgical attempts in the current series included marginal excision in 7 cases $(9.6 \%)$, wide tumor resection in 35 cases $(48 \%)$, re-wide resection in 26 cases $(35.6 \%)$ and primary amputation in 4 cases $(5.5 \%)$. Only 1 patient $(1.3 \%)$ was not operated due to co-morbidities. There were no cases of secondary amputations.

LSS was performed on all patients. Surgical margins were re-assessable for this study in only 50 cases $(68.4 \%)$ : negative microscopic margins of $>1 \mathrm{~cm}$ were achieved in 14 cases, negative microscopic margins of $\leq 1 \mathrm{~cm}$ were found in 17 cases and microscopically positive margins were noted in 19 cases.

RT was delivered to 62 patients $(82.3 \%)$ after the surgical removal of the primary tumor. RT was not given in AMSTS IIIB cases, even after removal of the primary tumor.

At a median follow-up time of 6 years, local recurrence was observed in $17.8 \%$ of patients and systemic recurrence in $35.6 \%$ (local-only 6.8; systemic-only, 24.6; combined $11 \%$ ).

Twenty-four patients $(32.8 \%)$ had pulmonary metastases. The approach to recurrent SS was tailored individually to each case and consisted (when feasible) of the surgical removal of the local recurrence and pulmonary metastases, systemic doxorubicin + ifosfamide chemotherapy (if applicable) or ILP and RT to the local tumor bed or skeletal metastases. Of note, all patients with local-only recurrence were rendered disease-free by the surgical removal of the recurrence followed by RT, while 3 also received doxorubicin-based chemotherapy.

Therapy-related complications occurred in $35 \%$ of the patients, including superficial infection in $8 \%$, deep infection in $12 \%$, motor neuropathy in $6 \%$, pathologic fracture in $3 \%$, prosthesis loosening in 3\% (a patient with a true intra-articular SS underwent extra-articular resection and total knee replacement) and vascular injury in 3\%. No chemotherapyrelated death was observed.

The 10-year LRFS rate is $78 \%$, while the 10 -year SRFS rate is $68 \%$. The median SRFS time is 180 months and the median LRFS has not yet been reached. The 10-year OS rate is $61 \%$. The median OS time has not yet been reached (Fig. 1).

Several prognostic factors were assessed in terms of local recurrence, systemic recurrence and OS in patients with SS: histology (biphasic vs. monophasic), gender (female vs. male), age ( $\leq 20$ vs. $>20$ years), no. of biopsies performed for diagnosis (1 vs. 2), limb (upper vs. lower), time to diagnosis ( $\leq 1$ vs. $>1$ year), mass-related symptoms (asymptomatic vs. symptomatic), T stage (T1 vs. T2), neo-adjuvant chemotherapy vs. none, degree of necrosis ( $\geq 90$ vs. $<90 \%$ ), ILP vs. none, resection type (R0 vs. R1), post-operative RT vs. none, and induction ILP vs. none. Induction therapy by ILP (TNF + melphalan) was superior to having none in terms of local control (HR, 0.2608; 95\% CI, 0.04010-0.8490; $\mathrm{P}=0.03$ ). Time to diagnosis of a year or less was superior to a delayed diagnosis in terms of systemic control (HR, 0.3354; 95\% CI, $0.1567-0.9457 ; \mathrm{P}=0.037)$. OS was not affected by any of the investigated parameters.

It should be noted that the analysis included only sarcomarelated events and that neoadjuvant therapy was administered in the more locally advanced cases (a selection bias). Age cut-off was chosen arbitrarily to discriminate between the first two decades and the rest of life. For many parameters the hazard ratio (HR) seemed to be reduced or increased, but was not statistically significant because of the short followup (a follow-up of 15 years or much more is probably required).

\section{Discussion}

Seventy-three consecutive patients with SS of the extremities and trunk, treated at one medical center by a multidisciplinary team and followed for more than 15 (median 6) years, constitute a relatively large, homogeneous and matured series. Our results point to several important conclusions regarding the clinical behavior of SS.

$\mathrm{SS}$ is a long-lasting disease with slow dynamics of recurrence and killing. The accumulation of local and systemic recurrence events following a limb sparing approach did not reach a plateau even 192 months after diagnosis. Consequently, LRFS and SRFS (distant metastases) curves tend to slope down over a very long period of time following definitive therapy. On the other hand, the OS curve of patients with SS reached a plateau after 7 years, with no subsequent sarcomarelated death events for a period of 10 years. The practical aspects of these observations are the need for long-term follow-up in order to diagnose recurrences, the fact that not all local or distant recurrences are necessarily associated with a shortening of the OS time and the need, in each case of recurrence, to individually tailor therapy. Other types of STS, such as liposarcoma (14), also necessitate a long-term followup because of late recurrences. In liposarcoma, a 5-year follow-up is definitely insufficient; at least 10 years of followup should be applied with biannual visits. In our experience (14), the time to failure curve did not reach a plateau even after 10 years of follow-up because of continued recurrences at this time-point. On the other hand, as reported specifically for MFH (16), 5 years sufficed for most of STSs (15) because late events were very uncommon.

Several prognostic factors affecting LRFS, SRFS and OS were investigated in this study. Their influence was expressed by HR. However, due to small numbers in certain subgroups and the low rate and slow dynamics of events in the whole series, the cohort of patients has not, despite the long followup, yet reached statistical maturation or yielded statistically significant $\mathrm{p}$-values. We believe that a longer follow-up period will yield significant differences in HR.

LRFS seemed to be better in cases of biphasic type, female gender, age under 20 years at diagnosis, one biopsy for establishing the diagnosis, lower limb tumors, shorter time to diagnosis, symptomatic mass, administration of preor post-operative chemotherapy (the bias in patient selection is explained earlier in this report), administration of preoperative ILP (and not pre-operative chemotherapy), R0 resection and administration of post-operative RT.

SRFS seemed to be better in cases of biphasic type, age under 20 years at diagnosis, one biopsy for establishing the diagnosis, shorter time to diagnosis, asymptomatic primary mass, T1 tumor, induction chemotherapy (and not induction ILP) and necrosis of more than $90 \%$ in response to preoperative chemotherapy (the bias in patient selection is explained earlier in this report).

OS seemed to be better in cases of biphasic type, age under 20 years at diagnosis, upper limb location, shorter time to 
diagnosis, asymptomatic mass, T1 tumor and administration of chemotherapy either palliative or curative.

Long lists of prognostic factors for local and systemic control of STS in general and SS in particular have been published in the literature. Since the patient populations varied from one series to another, the outcome of all statistical analyses has seemed to vary as well.

In a large but non-homogeneous series which focused on a variety of soft tissue sarcomas, the 5-year survival rate was $76 \%$ with a median follow-up time of only 4 years (17). Significant independent adverse prognostic factors for local recurrence were age over 50 years, recurrent disease at presentation and microscopically positive surgical margins; for distant recurrence they were intermediate tumor size, high histological grade and deep location. For diseasespecific survival, large tumor size, high-grade, deep location, recurrent disease at presentation, microscopically positive surgical margins and lower extremity site were adverse factors. For post-metastasis survival, only large tumor size $(>10 \mathrm{~cm})$ was an adverse prognostic factor (17).

In a smaller series than this which followed 48 patients with SS, tumor size of $<5 \mathrm{~cm}$ had a cancer-specific survival rate of $100 \%$ at 10 years, compared with 10 -year survival rates of 32 and $0 \%$ for those with sarcomas of $5-10 \mathrm{~cm}$ and $>10 \mathrm{~cm}$, respectively (5). Patients with SS with less than 10 mitoses per 10 high-power fields (HPF) had a 10-year cancer-specific survival rate of $46 \%$, compared with a 10 -year survival rate of $14 \%$ for those with sarcomas with greater than 10 mitoses per HPF. Patients with a clean margin of excision were found to have a 10 -year cancer-specific survival rate of $43 \%$, compared with $0 \%$ for those with microscopic positive margins $(\mathrm{P}=0.03)(5)$.

In a larger series which followed 112 patients with SS of the extremities, the median duration of follow-up was 72 months. The 5-year local recurrence, distant recurrence and mortality rates were 12,39 and $25 \%$, respectively. Tumor size of $\geq 5 \mathrm{~cm}$ and the presence of bone or neurovascular invasion were independent adverse predictors of distant recurrence and mortality (18).

The largest series to focus on SS to date included 271 patients of all age groups from the Rizzoli Center. The 5-year event-free survival rate for the study cohort as a whole was $37 \%$, although this rate varied with age. Among patients with surgically resected disease, the 5-year metastasis-free survival rate was $60 \%$ for those who were treated with chemotherapy and $48 \%$ for those who were not. Similar observations were also made in our series. In the subgroup of patients with measurable disease, the rate of tumor response to chemotherapy was approximately $48 \%$ (19).

The role of induction ILP with $\mathrm{TNF} \alpha$ as induction therapy has never been compared in a controlled randomized study. In the current series, the impacts of ILP versus induction chemotherapy on LRFS, SRFS and OS were assessed using a non-randomized method. Since the two procedures were used by the same team at one center according to the same multidisciplinary approach, and on a homogeneous population of patients with SS, this comparison was feasible. Our results clearly show that the LRFS, but not the SRFS or OS time, is significantly better for ILP-treated than for chemotherapytreated patients. Systemic chemotherapy, on the other hand, showed signs of improving the SRFS and OS, although the differences did not achieve statistical significance. It is conceivable that a combination of induction ILP and postoperative systemic chemotherapy may better control the disease, both locally and systemically. This point, however, needs to be further investigated in a prospective study.

In conclusion, we have showed that SS is a chronic disease with very late events of recurrence which do not necessarily affect the OS. Several prognostic factors for LRFS, SRFS and OS have been identified. Induction ILP with TNF $\alpha$ seems to be better for local control, supporting the idea of tailoring therapy according to the specific type of STS.

\section{References}

1. Fisher C: Synovial sarcoma. Ann Diagn Pathol 2: 401-421, 1998.

2. Enzinger FM and Weiss SW (eds): Benign tumors and tumorlike lesions of synovial tissue. In: Soft Tissue Tumors. 3rd edition. Mosby, St. Louis, MO, 1995.

3. Enzinger FM and Weiss SW (eds): Synovial sarcoma. In: Soft Tissue Tumors. 3rd edition. Mosby, St. Louis, MO, 1995.

4. Ordonez NG, Mahfouz SM and Mackay B: Synovial sarcoma: an immunohistochemical and ultrastructural study. Hum Pathol 21: 733-749, 1990.

5. Singer S, Baldini EH, Demetri GD, et al: Synovial sarcoma: prognostic significance of tumor size, margin of resection, and mitotic activity for survival. J Clin Oncol 14: 1201-1208, 1996.

6. Clark J, Rocques PJ, Crew AJ, et al: Identification of novel genes, SYT and SSX, involved in the $\mathrm{t}(\mathrm{X} ; 18)(\mathrm{p} 11.2 ; \mathrm{q} 11.2)$ translocation found in human synovial sarcoma. Nat Genet 7: 502-508, 1994.

7. O'Sullivan MJ, Kyriakos M, Zhu X, et al: Malignant peripheral nerve sheath tumors with $\mathrm{t}(\mathrm{X} ; 18)$. A pathologic and molecular genetic study. Mod Pathol 13: 1336-1346, 2000.

8. Storlazzi CT, Mertens F, Mandahl N, et al: A novel fusion gene, SS18L1/SSX1, in synovial sarcoma. Genes Chromosomes Cancer 37: 195-200, 2003.

9. Morrison C, Wakely PE Jr, Ashman CJ, et al: Cystic synovial sarcoma. Ann Diagn Pathol 5: 48-56, 2001.

10. Merimsky O, Meller I, Issakov J, et al: Adriamycin-ifosfamide induction chemotherapy for extremity soft tissue sarcoma: comparison of two non-randomized protocols. Oncol Rep 6: 913-920, 1999.

11. Gutman M, Inbar M, Lev-Shlush D, et al: High dose tumor necrosis factor-alpha and melphalan administered via isolated limb perfusion for advanced limb soft tissue sarcoma results in a $>90 \%$ response rate and limb preservation. Cancer 79: 1129$1137,1997$.

12. Enneking WF, Spanier SS and Goodman MA: A system for the surgical staging of musculoskeletal sarcoma. Clin Orthop Relat Res: 106-120, 1980.

13. Merimsky O, Soyfer V, Kovner F, et al: Limb sparing approach: adjuvant radiation therapy in adults with intermediate or highgrade limb soft tissue sarcoma. Radiother Oncol 77: 295-300, 2005 .

14. Issakov J, Soyfer V, Kollender Y, Bickels J, Meller I, Merimsky O: Liposarcoma in adult limbs treated by limbsparing surgery and adjuvant radiotherapy. J Bone Joint Surg Br 88: 1647-1651, 2006.

15. Leyvraz $\mathrm{S}$ and Jelic S: ESMO minimum clinical recommendations for diagnosis, treatment and follow-up of soft tissue sarcomas. Ann Oncol 16 (Suppl. 1): S69-S70, 2005.

16. Issakov J, Kollender Y, Soyfer V, et al: A single-team experience of limb sparing approach in adults with high-grade malignant fibrous histiocytoma. Oncol Rep 14: 1071-1076, 2005.

17. Pisters PW, Leung DH, Woodruff J, et al: Analysis of prognostic factors in 1,041 patients with localized soft tissue sarcomas of the extremities. J Clin Oncol 14: 1679-1689, 1996.

18. Lewis JJ, Antonescu CR, Leung DH, et al: Synovial sarcoma: a multivariate analysis of prognostic factors in 112 patients with primary localized tumors of the extremity. J Clin Oncol 18: 2087-2094, 2000.

19. Ferrari A, Gronchi A, Casanova M, et al: Synovial sarcoma: a retrospective analysis of 271 patients of all ages treated at a single institution. Cancer 101: 627-634, 2004. 